\title{
BIDENS PILOSA L. Y BIDENS ODORATA CAV. (ASTERACEAE: HELIANTHEAE) EN LA VEGETACION URBANA DE LA CIUDAD DE MEXICO
}

\author{
HeIKe ViBRAnS \\ Escuela de Ciencias, El Cerrillo \\ Universidad Autónoma del Estado de México \\ Instituto Literario Ote. No. 100 \\ 50000 Toluca, México \\ o: Apartado Postal 519, 50000 Toluca
}

\section{RESUMEN}

Bidens pilosa L., no citada en trabajos florísticos recientes, es un miembro común de la flora ruderal urbana en el Valle de México. Se discute su ecología, comparándola con la de Bidens odorata Cav.

\section{ABSTRACT}

Bidens pilosa L., not cited in recent floristic works, is found to be a common member of Mexico City's urban ruderal flora. Some aspects of its ecology are discussed and compared with those of Bidens odorata Cav.

Bidens pilosa es una maleza tropical de origen americano con una distribución amplia en el mundo; es una de las malas hierbas anuales más importantes sobre todo en América Latina y Africa Oriental (Holm et al., 1977).

Bidens odorata habita principalmente las cadenas montañosas de México, llegando al suroeste de los Estados Unidos y a Centroamérica (Ballard, 1986). Es una planta muy característica del paisaje del Altiplano transformado por el hombre, asociada sobre todo al cultivo de maíz.

Las dos especies pertenecen a un grupo de taxa cercanamente emparentados, cuya sistemática y taxonomía fue muy confusa durante mucho tiempo. Ballard $(1975,1986)$ lo aclaró parcialmente. El mencionado autor encontró tres especies (con variedades cada una): la diploide $(n=12)$ Bidens odorata Cav., la tetraploide B. alba (L.) DC $(n=24)$, y la hexaploide $B$. pilosa L. $(n=36)$. Los diferentes niveles de ploidía se correlacionaban con ciertos rasgos morfológicos. Su clasificación fue aceptada por Melchert (1976), McVaugh (1984) y Rzedowski \& Rzedowski (1985). Desafortunadamente Ballard (1986) sólo trata las variedades de Bidens odorata con claves y descripciones, mientras que las variedades de B. pilosa solamente se mencionan de paso.

Las referencias a $B$. pilosa en la literatura más antigua son ambiguas, ya que antes este nombre se usaba para todo el complejo. 
Bidens pilosa en la Ciudad de México

Durante nuestras investigaciones sobre la vegetación urbana en el centro de la Ciudad de México nos llamaron la atención unas plantas de Bidens con cabezuelas discoides, o sea, sin flores liguladas. La referencia al citado trabajo de Ballard (1986), complementado con recuentos de los cromosomas de 5 colectas y con uno de $B$. odorata como control, permitió identificarlas claramente como Bidens pilosa L. El conteo se llevó a cabo en el Laboratorio de Fanerogamia del Instituto de Biología, Universidad Nacional Autónoma de México. Se prepararon células mitóticas de puntas de raíces de aquenios recién germinados, pretratados con 8-hidroxiquinoleina y teñidos con la técnica de Feulgen.

En trabajos recientes se ha citado solamente a Bidens odorata del Valle de México (Rapoport et al., 1983; Rzedowski y Rzedowski, 1985; mapas de distribución en Ballard, 1986). En las colecciones del Herbario Nacional (MEXU), Universidad Nacional Autónoma de México, no se encontraron ejemplares del área en cuestión; las (relativamente pocas) colectas que se pueden referir a $B$. pilosa provienen todas de localidades ubicadas por debajo de 2000 m s.n.m.

Sin embargo, Paray (1957) menciona expresamente a Bidens pilosa con cabezuelas discoides y dice: "es esencialmente ruderal, encontrándola muchas veces en las calles de la capital". Aunque en su descripción de la especie indica "cabezuelas discoides o raras veces obscuramente radiadas, con las lígulas minúsculas, de 7-8 $\mathrm{mm}$ de ancho y 5-6 $\mathrm{mm}$ de alto", más abajo la ilustración correspondiente muestra plantas con flores liguladas largas. Esta confusión probablemente sólo refleja la que existió por mucho tiempo en el complejo. Pero, en mi opinión, Paray no hubiera mencionado a una Bidens discoide con detalles sobre su hábitat, si nunca la hubiera visto; así que se puede suponer que Bidens pilosa existe en la Ciudad de México desde hace por lo menos medio siglo, pero no ha sido colectada a causa de su hábitat ruderal (Paray no cita ejemplares).

Bidens pilosa se encuentra con una frecuencia alta en toda el área de investigación, entre el Bosque de Chapultepec y el Aeropuerto y entre el Campus del Instituto Politécnico Nacional en Lindavista y la Ciudad Universitaria. Como ejemplos se citan los siguientes ejemplares, todos en la Ciudad de México, de los cuales se investigó el número de cromosomas:

Delegación Benito Juárez, Colonia Alamos, Calle Aragón, esquina con Coruña. Lado SE. Camellón con árboles todavía muy pequeños. Con Pennisetum clandestinum, Bromus catharticus, 2.XIII.1993, Vibrans 4378. - Delegación Cuauhtémoc, Colonia Tlatelolco, Eje $2 \mathrm{~N}$, casi esquina con Eje 1. Lado NW, terreno atrás de fábrica abandonada. Con Pennisetum clandestinum, Sicyos deppei, Cynodon dactylon, 15.XIII.1993, Vibrans 4424. - Delegación Gustavo A. Madero, Colonia Nuevo Atzacoalco, terreno baldío entre Calle 329 y el Gran Canal. Con Pennisetum clandestinum, Eleusine multiflora, Malva parviflora, Atriplex suberecta, Aster subulatus, 16.XIII.1993, Vibrans 4442. - Delegación Iztapalapa, Colonia Guadalupe del Moral, terrenos baldíos al E de la salida de la Central de Abasto, 6.II.1994, Vibrans 4732. - Delegación Cuauhtémoc, Colonia Algarín, Calle Isabel la Católica, entre Calle J. E. Hernández y Calle Medina, camellón con árboles y arbustos, con Chenopodium murale, Eleusine indica, Taraxacum officinale, 11.Il.1994, Vibrans 4761. Los ejemplares citados se encuentran depositados en MEXU.

Llama la atención que $B$. pilosa también crece y florece en invierno. 
Vibrans: Bidens pilosa y Bidens odorata en la Vegetación Urbana de la Ciudad de México

Taxonomía

Según Ballard (1986), B. pilosa var. pilosa, la única variedad que cita de México, se distingue de $B$. odorata por tener

- cabezuelas discoides o con lígulas muy cortas y entonces con aquenios fértiles,

- generalmente más flores (25-40) por cabezuela,

- un vilano de 3-5 aristas retrorsamente barbadas en aquenios maduros (en $B$. odorata son 2 o ninguna, a veces barbados y a veces no).

- $2 n=72$ cromosomas (B. odorata tiene $2 n=24)$.

Normalmente las hojas son muy parecidas, consistiendo de 3-7 folíolos más o menos ovados y aserrados; en $B$. odorata existen también individuos con hojas muy partidas.

De estas diferencias sólo el número cromosómico constituye un criterio absoluto. Es posible encontrar Bidens pilosa con flores liguladas y lígulas casi indistinguibles de las de $B$. odorata y sólo algunos aquenios con vilano de 3 ó 4 aristas (var. minor?). Además, las ilustraciones de Bidens pilosa, p.ej. de Cuba (Hadac y Hadacová, 1968) serían identificadas morfológicamente como $B$. odorata según los criterios enlistados arriba, pero B. odorata no ha sido citada para Cuba hasta ahora. Asimismo, Capote et al. (1986) encontraron que la proporción de aquenios con 3 o más aristas puede depender del estrés hídrico de la planta. El número de flores por cabezuela tampoco es un criterio muy confiable.

A pesar de las dificultades en la identificación de algunos individuos, es claro que existen dos grupos de plantas en la Ciudad de México, cuyos miembros se pueden separar en por lo menos $95 \%$ de los casos basándose en criterios morfológicos. Ya que existe además la mencionada diferencia en niveles de ploidía (así como divergencias en su ecología, discutidas abajo), está bien fundada la separación en dos especies. Por otro lado sin duda hace falta una investigación taxonómica más profunda, sobre todo de Bidens pilosa L. S.str.

\section{Ecología}

Se observó una diferencia entre el hábitat de las dos especies: B. odorata aparece principalmente en áreas medianamente alteradas y más abiertas, p.ej. los alrededores de la Ciudad Universitaria, en panteones o terrenos baldíos; mientras que B. pilosa se encuentra a menudo en lugares fuertemente perturbados y delimitados, como pies de árboles, camellones y grietas. Tal disparidad no es absoluta, pues a veces se pueden encontrar ambas especies lado a lado.

Este comportamiento se explica fácilmente en función del sistema de polinización ya que según Ballard (1986), B. odorata es auto-incompatible -y por lo tanto necesita mantener cierto tamaño de población- mientras que $B$. pilosa es auto-compatible y puede mantener poblaciones de 1 ó 2 individuos por varios años.

Otros factores de importancia son el clima y las diferencias en los requerimientos para la germinación. No existen estudios comparativos relativos a estos aspectos, sólo trabajos dispersos de varios países. 
Según Hadac y Hadacová (1968), B. pilosa germina bien en Cuba bajo luz alternada y temperaturas fluctuando entre 27 y $29^{\circ} \mathrm{C}$. Reddy \& Singh (1992) encontraron un intervalo ideal para el fenómeno en esta especie de $25 / 20$ o 35/30 $\mathrm{C}$ (fluctuación día/noche), mientras que con temperaturas abajo de $15 / 10^{\circ} \mathrm{C}$ disminuye la cantidad de plántulas obtenidas.

Corkidi et al. (1991) estudiaron el comportamiento de las semillas de Bidens odorata, provenientes de una localidad relativamente caliente (temperatura media anual de $26^{\circ} \mathrm{C}$.). Germinaron menos aquenios en un régimen de temperatura que fluctuó de $25 \% 35^{\circ}$ que en uno de $15^{\circ} / 25^{\circ}$. La intensidad más alta se obtuvo con una temperatura constante de $25^{\circ} \mathrm{C}$.

Olmos y Silvestre (1988) encontraron que Bidens odorata germina en proporciones más altas en primavera que en verano. Ocampo (1987) informó que en "Bidens pilosa", colectada cerca de Chapingo, Méx., aproximadamente a 2300 m s.n.m. el proceso se realiza mucho mejor a temperaturas inferiores a $15^{\circ} \mathrm{C}$; ; pero es probable que en este último caso se trata de $B$. odorata.

De lo anterior puede deducirse que posiblemente hay una diferencia en las temperaturas óptimas para la germinación entre las dos especies. Lo anterior explicaría el hecho de que Bidens pilosa no existe en las áreas con cultivos alrededor de la Ciudad de México. Cabe recordar que la temperatura promedio en la zona urbana de la capital de la República está bastante por encima de la correspondiente a superficies sin edificar, como es el caso en todas las metrópolis grandes.

Es probable que estos dos factores -sistema de polinización y el clima urbano- hayan permitido que una planta básicamente termófila se estableciera en una región de clima templado, más allá de su intervalo normal de altitud.

\section{AGRADECIMIENTOS}

El proyecto "Vegetación ruderal de la Ciudad de México" es un subproyecto de la línea de investigación "Estudios ecológicos en especies nativas e introducidas de la flora espontánea del Valle de México" (IN-207892 DGAPA, UNAM). Agradezco el apoyo de los responsables del proyecto, Dra. Alma Orozco y Dr. Robert Bye. La Escuela de Ciencias, Universidad Autónoma del Estado de México, me prestó toda la ayuda necesaria. El Dr. Fernando Chiang leyó y corrigió el manuscrito.

\section{LITERATURA CITADA}

Ballard, R. 1975. A biosystematic and chemotaxonomic study of the Bidens pilosa complex in North and Central America. Ph. D. Diss., Univ. of lowa, lowa City. (Cit. en Melchert, 1976).

Ballard, R. 1986. Bidens pilosa complex (Asteraceae) in North and Central America. Amer. J. Bot. 73(10): $1452-1465$.

Capote, S., R. Orta y E. Pérez. 1986. Estrategia de reproducción de una mala hierba: Bidens pilosa L. Rev. Jard. Bot. Nac. (Habana) 7(1): 73-79.

Corkidi, L., E. Rincón y C. Vázquez-Yanes. 1991. Effects of light and temperature on germination of heteromorphic achenes of Bidens odorata (Asteraceae). Can. J. Bot. 69: 574-579.

Hadac, E. y V. Hadacová. 1968. Contribución a la ecología de Bidens pilosa L. en Cuba. Acad. Cienc. Cuba, Serie Biol., No. 2. 14 pp. 
Holm, L. G., D. L. Plucknett, J. V. Pancho y J. P. Herberger. 1977. The world's worst weeds. Distribution and ecology. University Press. Honolulu. 597 pp.

McVaugh, R. 1984. Flora Novo-Galiciana. A descriptive account of the vascular plants of Western Mexico. Compositae. Vol. 12. University of Michigan Press. Ann Arbor. 1161 pp.

Melchert, T. E. 1976. Bidens, en Flora of Guatemala. Fieldiana Bot. 24(12): 193-214.

Ocampo R., R. A. 1987. Influencia de la temperatura, luz, estratificación y escarificación mecánica sobre la germinación de cuatro especies de malezas de importancia agrícola en México. Tesis profesional. Universidad Autónoma de Chapingo. México. 79 pp.

Olmos M., I. y B. V. Silvestre. 1988. Contribución al estudio de la germinación de semillas de diez especies de malas hierbas. Memoria, IX Congreso Nacional de la Ciencia de la Maleza, 2628 Oct. de 1988, Cd. Juárez, Chihuahua. pp. 39-49.

Paray, L. 1957. El género Bidens en el Valle de México. Bol. Soc. Bot. Méx. 20: 1-12.

Rapoport, E. H., M. E. Díaz Betancourt e I. R. López Moreno. 1983. Aspectos de la ecología urbana en la ciudad de México. Flora de calles y baldíos. Limusa, México, D.F. 197 pp.

Reddy, D. N. y M. Singh. 1992. Germination and emergence of hairy beggar ticks (Bidens pilosa). Weed Science 40(2): 195-199.

Rzedowski, J. y G. C. Calderón de Rzedowski. 1985. Flora fanerogámica del Valle de México, Vol. 2. Inst. de Ecología, México, D.F. 674 pp. 